\title{
Application of Digital Surveying and Mapping Technology in Engineering Survey at This Stage
}

\author{
Wenjuan bai ${ }^{1, a}$ \\ ${ }^{1}$ Chongqing Vocational Institute of Engineering, Chongqing, China, 402260 \\ ${ }^{\mathrm{a} e m a i l,}$
}

Keywords: At This Stage; Engineering Survey; Digital Mapping Technology; Features; Application

\begin{abstract}
With the rapid development of social economy, Chinese urbanization and industrial construction process greatly accelerated, which in improving people's living standards and quality, but also on the construction of a higher demand. In the early work of engineering construction, it is necessary to carry out comprehensive measurement on all aspects. The measured data can provide reference for engineering design, so the accuracy of measurement data will have a great impact on the quality of construction. At present, in the engineering survey, people have gradually strengthened the application of digital mapping technology, which greatly solved the shortcomings and shortcomings in the traditional engineering measurement, which has an important effect on the improvement of engineering measurement efficiency. This paper mainly analyzes and studies the digital mapping technology and the application of digital mapping technology in engineering survey at present. It provides a reference for the digital surveying and mapping technology to get better application in engineering survey.
\end{abstract}

\section{Introduction}

Digital mapping technology has always been an important part of engineering measurement, mapping technology defects and deficiencies will have a direct impact on the quality of construction and construction quality, but also affect the late construction progress. In recent years, with the further development of science and technology, engineering measurement has gradually begun to use a variety of more advanced digital mapping technology, and the advantages of digital mapping technology has been fully convex, engineering measurement process is effective and efficient operation work patterns and modern people's work more adaptable rhythm. For example, in the engineering survey, the need to draw the corresponding drawings and topographic maps, in order to ensure that the proportion of topographic map is moderate, the survey staff will need to complete a lot of visits before drawing work to ensure the integrity and comprehensive data collection, After the collection is complete, you also need to spend a lot of time to calculate it, which will cause a waste of time. In addition, the use of digital mapping technology can also be stored in the information database related to the detailed for the latter part of the construction to provide convenience.

\section{The Overview of Digital Mapping Technology}

The Advantages of the Characteristics. The data measurement accuracy is high. Compared with the traditional measurement technology, the digital mapping technology in the specific measurement process can reduce the measurement error to the maximum, to maximize the accuracy of the measurement data. For example, assuming that the vertical distance of the graph is within the range of $300 \mathrm{~m}$, the position measurement error of the object to be measured needs to be controlled within $( \pm 3) \mathrm{mm}$. At the same time in the production of graphics and data transmission process, the data in the accuracy of almost no error, especially in the exhibition type, direction, line of sight and other common types of soil testing, the accuracy error is not there, this is to a large extent to ensure the accuracy of the final measurement results [1].

A high degree of automation. Digital mapping technology is essentially an intelligent technology, can fully demonstrate the computer data calculation function, while the use of the process can also 
be timely to determine whether the information is correct or not. Generally in the graphics drawing process need to use the corresponding icon symbols, and the use of digital mapping technology to draw the graphics will be more standardized and beautiful. Computer graphics are more sensitive to the existence of digital error problems. Even if the error is small, computer graphics can be effectively identified and corrected, which is important for the reduction of drawing error rate.

Graphic attributes rich psychological. The use of digital mapping technology not only can accurately mark the graphics of the coordinates of the information, while Kazakhstan can complete the display of all the points of the data information. Through the effective integration of the mapping point of the encoded information, and complete the mapping operation, and then the mapping system of the symbols, graphics can be used directly, but also can be used directly at any time using the corresponding symbol graphics, and then draw the final figure [2] In general, in the process of drawing the digital mapping technology, we can integrate all the data of the connection information, location information and attribute information of the topographic map, and the related attribute information of the graph can be perfected continuously.

It can fully use GIS information source. Digital mapping technology can provide GIS (geographic information system) to provide a large amount of data information, to further optimize and improve the geographic information system data. At this stage, with the gradual improvement of the geographic information system and improved digital mapping technology, between the two can be a very good seamless docking, but with the continuous development and innovation of digital mapping technology, in the next period of time it will be able to fully agree with the geographic information system, integration. At present, the integration between the two is mainly reflected in the following aspects: First, the engineering measurement location in the wild when the digital mapping technology can be measured with the geographic information system database with the relevant measurement data and source data for comparison In order to be able to timely detect the existence of defects and shortcomings, effective correction; Second, the current digital measurement technology in the land cadastral, urban planning and other projects to obtain data information, the geographic information system stored in the relevant data can be fully utilized [3].

It is conducive to graphics editing. In the process of using digital mapping technology, if the data is known, then it will be applied to hierarchical placement of its processing and analysis, which is mainly due to the use of hierarchical placement can be the amount of surface expansion, and later, if you need to process or use the map information, it will be more convenient. For example, a housing construction due to the actual needs of the need for its expansion or transformation, so in the expansion or renovation process will change the cadastral information, if you want to complete the system in the complete entry of new information, then it needs re-integration, processing the original relevant data, and the use of hierarchical placement can automatically integrate the original information and new information, so a good guarantee of data integrity and timeliness.

The Important Significance. In recent years, engineering applications in the digital mapping technology is more widely used, and the development of fast, in the original engineering surveying and mapping process, not only need to spend a lot of manpower, material and financial resources, while the entire mapping process due to the data is very large, complicated, and then prone to data errors, omissions and measurement errors and other issues, the adverse effects of the late construction. And through the use of digital mapping technology, on the one hand to ensure the accuracy of measurement results, while dealing with digital computing and drawing time are greatly reduced, human, material, financial waste and construction time are effectively saved [4]. Therefore, the application of digital mapping technology in the engineering survey is to improve the efficiency of the same time, the accuracy of data measurement has also been effectively guaranteed to avoid serious waste of various resources.

\section{The Application of Digital Mapping Technology in Engineering Measurement at the Current Stage}

The Applications of 35 Technology in the Engineering Measurement. GPS. As a positioning system, the accuracy of GPS is relatively high, but also in the engineering survey will not be 
weather, location, time and other conditions, do not need the help of the corresponding auxiliary equipment, the final mapping of accurate data The degree is relatively high. GPS in the process of engineering measurement, the direct use of dynamic pile stakeout, while the pile position obtained by the lofting position is more accurate, due to various measurement conditions are limited, so the efficiency of measurement work has been greatly improved [5]. Also in the specific operation, you can also directly check the center of the pile, to avoid the pile surface centrifugal problems, while the construction efficiency can also be a very good guarantee.

GIS. In GIS technology, the application of various disciplines is more, the use of computer technology can effectively collect and organize the relevant data, and to achieve visual management and automatic storage of measurement data, widely used in land planning, urban management and other fields. GIS technology can also vector analysis of known things or objects, the use of data collected directly to the corresponding graphics, and then intuitive presentation of the information data to ensure that information can be quickly extracted data to facilitate the construction, but also conducive to long-term planning.

RS. RS technology has a large area of synchronous observation function, in the application of engineering measurement can be dynamic information on the collection, compared to the comprehensive processing of information and information differences have certain advantages. As a commonly used geographic information observation tool, RS technology coverage range is relatively wide, but also can use the spectral resolution technology to obtain the corresponding terrain change information, in the cadastral map and urban topographic map has a positive role, so in engineering measurement Can be further promoted and applied [6].

The Applications of Ground Digital Mapping Technology in the Engineering Survey. If the scale of the map is relatively large, the application of terrestrial digital mapping technology is more extensive. Through the integrated operation flow of collecting, collating, drawing and outputting related information, the accuracy of data mapping can be further improved. The application of ground digital mapping technology is also more. In the engineering measurement through the specific application, can be directly based on the measured results drawn a different proportion of the map to ensure that the construction needs can be well met, effectively avoid duplication of work. At the same time in the process of collecting data, digital mapping technology in the three-point positioning project can also measure the data within the scope of automatic collection, storage, processing analysis, this integrated operation process effectively avoid the human factors caused by data error can significantly improve the mapping, drawing and other drawing accuracy [7]. In addition, the digital results can also be stored in the computer equipment for a long time, as long as there is no damage, at any time can be used at any time, and drop the data drawn into the relevant graphics, its intuitive greatly improved, but also for the construction of reference Basis, but also can use CAD technology to deal with data composition map information, to achieve accurate reading of the distance between the graphics point and coordinate information.

The Applications of Original Digital Mapping Technology in the Engineering Measurement. According to hand tracking, vector graphics, GPS input to digitize the original graphics and data and you can greatly improve the visuality of the graphics and clarity, but also to facilitate the late changes. In the actual process of dealing with the need to be in accordance with national standards to determine the accuracy of cadastral maps and map scale, and then to ensure that the specific project in the red to fully play the role of drawing. Which in the original digital mapping, in order to ensure the accuracy of mapping results, in the formal mapping before the need to debug equipment to ensure that the normal operation of the equipment, but also need to protect the accuracy of manual tracking, it will have a decisive impact on the original processing accuracy. It is important to note that the work attitude and professional level of surveying and mapping personnel play an important role in the high-precision mapping of digital figures, thus strengthening the education and training of surveying and mapping personnel, focusing on the improvement of its comprehensive quality and professional level, which will directly determine the measurement data accuracy [8]. But also need to follow the relevant provisions to organize data and hierarchical coding processing data, and then generate digital graphics. 
The Application of Digital City in Engineering Survey. Digital city is a new type of engineering measurement, which is characterized by large scale, complex procedures, involving a wide range, so it has a strong comprehensive. But also in the digital city, the requirements for science and technology is relatively high, in the process of economic integration in the process of the need for the full application of computer technology, and then the urban geographic coordinates to build a complete and unified framework, and store important information, And to promote the further improvement of social information, when the latter need to use a certain aspect of the information, you can directly in the system through the retrieval [9].

\section{Conclusion}

In general, the further development of science and technology in the engineering survey in the better application of digital mapping technology provides technical support, and the use of digital mapping technology, but also can greatly improve the integrity and accuracy of engineering measurements for the late Construction to provide more comprehensive, complete and intuitive information data to ensure the quality of construction projects to meet the expected standards. At present, the digital mapping technology used in engineering measurement mainly includes $3 \mathrm{~S}$ technology, terrestrial digital mapping technology, digital mapping technology and several aspects of digital city, and with the continuous optimization and improvement of science and technology, these technologies in engineering measurement In the effective role can be effectively played, and there will be more advanced technology in the application of engineering applications. Through the application of digital mapping technology, engineering projects can greatly shorten the measurement cycle and improve work efficiency to facilitate social construction.

\section{References}

[1] Zhang wenling. Application of Digital Mapping Technology in Engineering Survey[J / OL]. Engineering Technology Research, 2017, (03): 78 + 102. (2017-03-24)

[2] Song Zengxiao, Yan Lixiang.Application of Digital Surveying and Mapping Technology in Engineering Survey [J / OL]. West Resources, 2016, (05): 133-134. (2016-11-01)

[3] Zhang Yongqing. Digital mapping technology in the application of engineering measurement[J]. Chinese new technology and new products, 2016, (15): 114-115.

[4] Ding Ming, Cui Shan Shan. Digital mapping technology in engineering measurement application research[J]. Shandong Industrial Technology, 2016, (11): 109.

[5] Wei Zhonggang. Digital mapping technology in the construction engineering survey application[J]. Shandong Industrial Technology, 2016, (08): 103. [

[6] Li Zhuo. Digital mapping technology in geological engineering survey application analysis[J]. Science and Technology Innovation Guide, 2015, 12 (30): 105-106.

[7] Luo Caiqing, He Jin. Digital mapping technology in engineering measurement application[J]. Science and Technology Innovation and Application, 2015, (05): 194.

[8] Li Bo, Yang Xiujie.Application of digital mapping technology in engineering survey [J]. Science and Technology Communications, 2013, 5 (09): 198 +196.

[9] Jiang Zhen, Zhou Yawen. Application of Digital Surveying and Mapping Technology in Engineering Survey[J]. Journal of Chifeng University (Natural Science Edition), 2012, 28 (15): 78-79. 